\title{
Analysis and Transformation in an Interactive Parallel Programming Tool *
}

\author{
Ken Kennedy Kathryn S. Mckinley Chau-Wen Tseng \\ ken@cs.rice.eduｋats@cri.ensmp.fr tseng@cs.rice.edu \\ Department of Computer Science \\ Rice University \\ Houston, TX 77251-1892
}

\begin{abstract}
The ParaScope Editor is a new kind of interactive parallel programming tool for developing scientific Fortran programs. It assists the knowledgeable user by displaying the results of sophisticated program analyses and by providing editing and a set of powerful interactive transformations. After an edit or parallelism-enhancing transformation, the ParaScope Editor incrementally updates both the analyses and source quickly. This paper describes the underlying implementation of the ParaScope Editor, paying particular attention to the analysis and representation of dependence information and its reconstruction after changes to the program.
\end{abstract}

\section{Introduction}

The ParaScope Editor is a tool designed to help skilled users interactively transform a sequential Fortran 77 program into a parallel program with explicit parallel constructs, such as those in PCF Fortran [35]. In a language like PCF Fortran, the principal mechanism for the introduction of parallelism is the parallel loop, which specifies that its iterations may be run in parallel according to any schedule. The fundamental problem introduced by such languages is the possibility of nondeterministic execution. Nondeterminism may occur if two different iterations of a parallel loop both reference the same memory location, where at least one of the references writes to the location. For example, consider converting the following sequential loop into a parallel loop.

$$
\begin{aligned}
& \text { DO I }=1,100 \\
& \mathrm{~A}(\mathrm{I})=\mathrm{A}(50)+1 \\
& \text { ENDDO }
\end{aligned}
$$

Because the parallel loop does not order its iterations, the value of each A(I) will depend on how early iteration 50 executes in the parallel execution schedule. Hence, the results may differ each time the program is executed. This kind of anomaly, often called a data race, precludes the parallelization of the above loop. In the literature of compilation for parallel execution, a potential data race is referred to as a loop-carried dependence [3, 34]. Without explicit synchronization, only loops with no carried dependences may be safely executed in parallel.

Automatic parallelizers use this principle by constructing a dependence graph for the entire program and then parallelizing every loop that does not carry a dependence. Unfortunately, a parallelizer is often forced

\footnotetext{
${ }^{*}$ This research was supported by the Center for Research on Parallel Computation (CRPC), a National Science Foundation Science and Technology Center. CRPC is funded by NSF through Cooperative Agreement Number CCR-9120008. Additional support was provided by IBM Corporation.
} 
to make conservative assumptions about whether dependences exist because of complex subscripts or the use of unknown symbolics. As a result, automatic systems miss many loops that could be parallelized. This weakness has led most researchers to conclude that automatic systems, by themselves, are not powerful enough to find all of the parallelism in a program.

However, the analysis performed by automatic systems can be extremely useful to the programmer during the parallelization process. The ParaScope Editor (PED) is based upon this observation. It is designed to support an interactive parallelization process in which the user examines a particular loop and its dependences. To safely parallelize a loop, the user must either determine that each dependence shown is not valid (because of some conservative assumption made by the system), or transform the loop to eliminate valid dependences. After each transformation, PED reconstructs the dependence graph so that the user may determine the level of success achieved and apply additional transformations if desired.

Clearly a tool with this much functionality is bound to be complex. PED incorporates a complete source editor and supports dependence analysis, dependence display, and a large variety of program transformations to enhance parallelism. Previous work has described the usage and motivation of the ParaScope Editor [6, 23, 32] and the ParaScope parallel programming environment [16]. In this paper, we focus on the implementation of PED's analysis and transformation features. Particular attention is paid to the representation of dependences, the construction of the dependence graph, and how dependences are used and incrementally reconstructed for each program transformation in an efficient and flexible manner.

We begin in Section 2 with a brief overview of dependence. Section 3 describes our work model. Section 4 presents PED's internal representations, and Section 5 examines the analysis strategy and algorithms in PED. Sections 6 and 7 explain PED's support for transformations and details an interesting subset. Section 8 summarizes strategies for updates following program and dependence edits. We conclude with a discussion of PED's modular design and related work.

\section{Dependence}

At the core of PED is its ability to analyze dependences in a program. Dependences describe a partial order between statements that must be maintained to preserve the meaning of the original sequential program. A dependence between statement $S_{1}$ and $S_{2}$, denoted $S_{1} \delta S_{2}$, indicates that $S_{1}$, the source, must be executed before $S_{2}$, the sink. There are two types of dependence, data and control dependence, which are described below.

\subsection{Data Dependence}

A data dependence, $S_{1} \delta S_{2}$, indicates that $S_{1}$ and $S_{2}$ read or write a common memory location in a way that requires their execution order to be preserved. There are four types of data dependence [34]:

True (flow) dependence occurs when $S_{1}$ writes a memory location that $S_{2}$ later reads.

Anti dependence occurs when $S_{1}$ reads a memory location that $S_{2}$ later writes.

Output dependence occurs when $S_{1}$ writes a memory location that $S_{2}$ later writes.

Input dependence occurs when $S_{1}$ reads a memory location that $S_{2}$ later reads. ${ }^{1}$

\footnotetext{
${ }^{1}$ Input dependences do not restrict statement order.
} 


\subsection{Control Dependence}

Intuitively, a control dependence, $S_{1} \delta_{c} S_{2}$, indicates that the execution of $S_{1}$ directly determines whether $S_{2}$ will be executed. The following formal definitions of control dependence and the postdominance relation are taken from the literature [19].

Def: $x$ is postdominated by $y$ in the control flow graph $\mathrm{G}_{f}$, if every path from $x$ to STOP contains $y$, where STOP is the exit node of $\mathrm{G}_{f}$.

Def: Given two statements $x, y \in \mathrm{G}_{f}, y$ is control dependent on $x$ if and only if:

1. $\exists$ a non-null path $p$, from $x$ to $y$, such that $y$ postdominates every node between $x$ and $y$ on $p$, and

2. $y$ does not postdominate $x$.

\subsection{Loop-Carried and Loop-Independent Dependence}

Dependences are also characterized as either being loop-carried or loop-independent [3]. Consider the following loop:

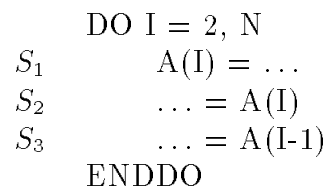

The true dependence $S_{1} \delta S_{2}$ is loop-independent because it exists regardless of the surrounding loops. Loopindependent dependences, whether data or control, occur within a single iteration of the loop and do not inhibit a loop from running in parallel. For example, if $S_{1} \delta S_{2}$ were the only dependence in the loop, this loop could be run in parallel, because statements executed on each iteration only affect other statements in the same iteration and not in any other iterations. However, loop-independent dependences do affect statement order within a loop iteration. Interchanging statements $S_{1}$ and $S_{2}$ violates the loop-independent dependence and changes the meaning of the program.

By comparison, the true dependence $S_{1} \delta S_{3}$ is loop-carried because the source and sink of the dependence occur on different iterations of the loop: $S_{3}$ reads a memory location that was written to by $S_{1}$ on the previous iteration. Loop-carried dependences are important because they inhibit loops from executing in parallel without synchronization. When there are nested loops, the level of any carried dependence is the outermost loop on which it first arises [3].

\subsection{Dependence Testing}

Determining the existence of data dependence between array references is more difficult than for scalars, because the subscript expressions must be considered. The process of differentiating between two subscripted references in a loop nest is called dependence testing. To illustrate, consider the problem of determining whether or not there exists a dependence from statement $S_{1}$ to $S_{2}$ in the following loop nest:

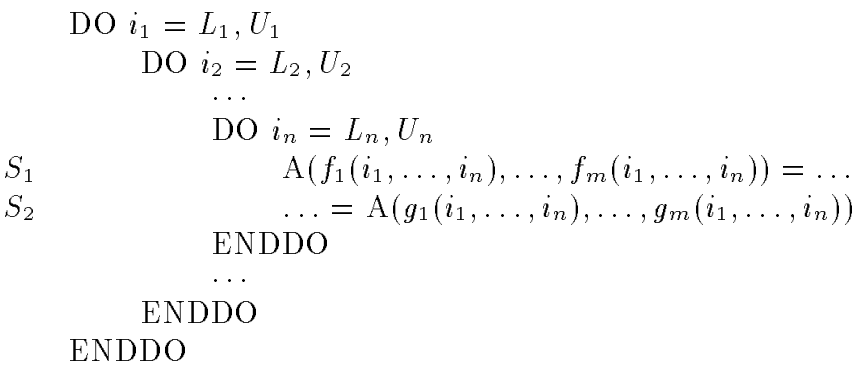


Let $\alpha$ and $\beta$ be vectors of $n$ integer indices within the ranges of the upper and lower bounds of the $n$ loops. There is a dependence from $S_{1}$ to $S_{2}$ if and only if there exist $\alpha$ and $\beta$ such that $\alpha$ is lexicographically less than or equal to $\beta$ and the following system of dependence equations is satisfied:

$$
f_{k}(\alpha)=g_{k}(\beta) \forall k, 1 \leq k \leq m
$$

\subsection{Distance and Direction Vectors}

Distance and direction vectors may be used to characterize data dependences by their access pattern between loop iterations. If there exists a data dependence for $\alpha=\left(\alpha_{1}, \ldots, \alpha_{n}\right)$ and $\beta=\left(\beta_{1}, \ldots, \beta_{n}\right)$, then the distance vector $\mathbf{D}=\left(D_{1}, \ldots, D_{n}\right)$ is defined as $\beta-\alpha$. The direction vector $\mathbf{d}=\left(d_{1}, \ldots, d_{n}\right)$ of the dependence is defined by the equation:

$$
d_{i}=\left\{\begin{array}{l}
<\text { if } \alpha_{i}<\beta_{i} \\
=\text { if } \alpha_{i}=\beta_{i} \\
>\text { if } \alpha_{i}>\beta_{i}
\end{array}\right.
$$

The elements are always displayed left to right, from the outermost to the innermost loop in the nest. For example, consider the following loop nest:

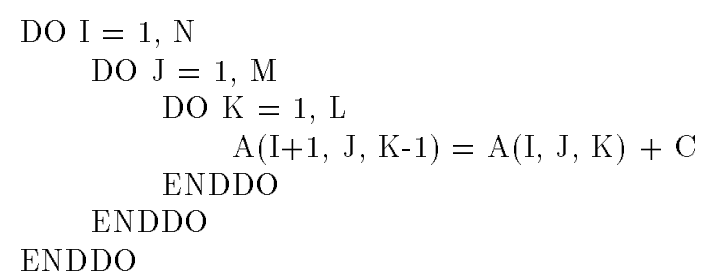

The distance and direction vectors for the true dependence between the definition and use of array A are $(1,0,-1)$ and $(<,=,>)$, respectively. Since several different values of $\alpha$ and $\beta$ may satisfy the dependence equations, a set of distance and direction vectors may be needed to completely describe the dependences arising between a pair of array references.

Direction vectors, introduced by Wolfe [48], are useful for calculating loop-carried dependences. A dependence is carried by the outermost loop for which the element in the direction vector is not an '='. Additionally, direction vectors are used to determine the safety and profitability of loop interchange $[3,48]$. Distance vectors are more precise versions of direction vectors that specify the actual number of loop iterations between two accesses to the same memory location $[3,48]$. They are employed by transformations to exploit parallelism and the memory hierarchy.

\section{Work Model}

PED is designed to exploit loop-level parallelism, which comprises most of the usable parallelism in scientific codes when synchronization costs are considered [15]. In the work model best supported by PED, the user first selects a loop for parallelization. PED then displays all of its carried dependences. The user may sort or filter the dependences, as well as edit and delete dependences that are due to overly conservative dependence analysis. PED also provides a set of intelligent program transformations that can be used to eliminate dependences. 


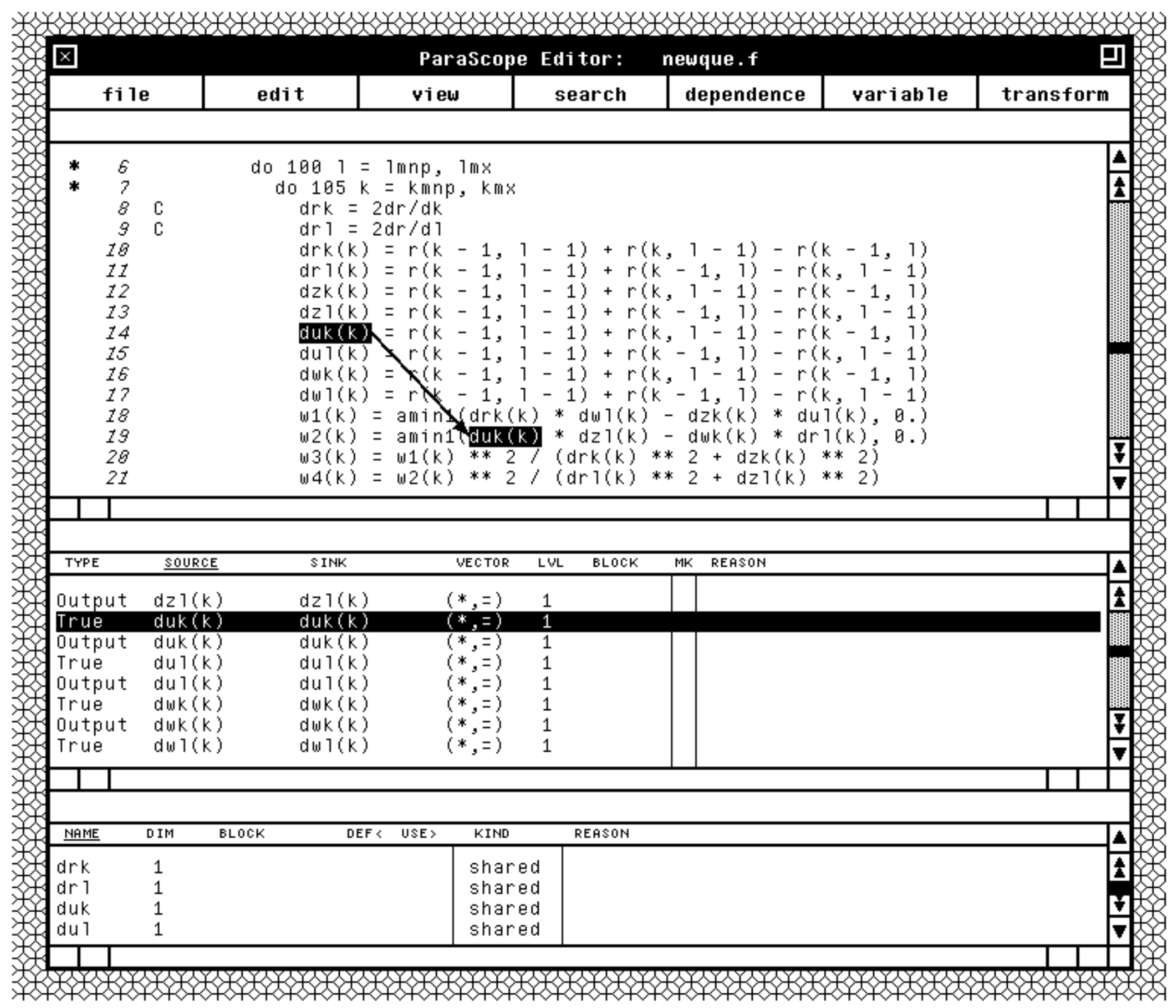

Figure 1: PED User Interface 
PED's user interface is shown in Figure 1. The top of the window is the program pane, which displays a loop from the subroutine newque in SIMPLE. ${ }^{2}$ The outer 1 loop is selected, which causes its header to be highlighted in a different color (not visible in the black and white picture). When a loop is selected, its carried dependences (and their characteristics) are exhibited in the middle dependence pane. In Figure 1 the true dependence involving variable duk is selected, causing it to be emboldened in the dependence pane. The dependence is simultaneously shown in the program pane with an arrow from its source to its sink. The variable pane at the bottom of the window discloses the private and shared status of the variables in the selected loop (see Section 5.3). Buttons across the top of each pane invoke various PED features, such as transformations and program analysis.

\section{Internal Representations}

This section describes the three major internal representations used by PED: the abstract syntax tree and its associated structures, the dependence graph, and loop information.

\subsection{Abstract Syntax Tree}

In PED and throughout the ParaScope programming environment, the program is represented using an abstract syntax tree (AST). Because the AST is a public structure, PED does not change the structure of an AST node nor use any fields within the AST. However, PED obviously needs to associate information such as data and control dependences with elements of the AST. The AST therefore has pointers to an associated structure, called a side array.

PED uses the side array to hold pointers into a variable sized info array. Elements of the info array hold pointers to information PED associates with AST nodes such as level vectors, reference lists, loop information, and subscript text. They also point to shadow expressions containing the results of symbolic analysis (discussed in Section 5.4). These structures are described in full below and illustrated for a sample program in Figure 2.

\subsection{Dependence Graph}

PED uses a statement dependence graph to represent control and data dependences in the program. The dependence graph is made up of dependence edges, which are connected using level vectors and reference lists. The level vectors provide the means for quickly collecting dependence edges on a statement pertaining to a specific loop level, and the reference lists provide the means for finding all the dependences associated with a particular variable reference.

\subsubsection{Dependence Edges}

Each data and control dependence in the program is represented as an explicit edge in the dependence graph. Dependences between pairs of variable references are reference level dependences. Statement level dependences arise due to input and output statements, branches out of loops, unanalyzed subroutine calls, and control dependences. An edge in the dependence graph is a data structure that describes the following significant features of the dependence.

- Type of the dependence: true, anti, output, input, control, i/o, exit, or call.

- Level of the loop carrying the dependence.

\footnotetext{
${ }^{2}$ SIMPLE is a two dimensional Lagrangian hydrodynamics program with heat diffusion produced by Lawrence Livermore National Laboratory
} 
- AST pointers for source/sink statements or references.

- Pointers to the source/sink level vectors and reference lists.

- Pointer to a hybrid direction/distance vector.

- Interchange-preventing and interchange-sensitive flags indicate the safety and profitability of loop interchange.

PED uses a hybrid direction/distance vector to store the results of dependence testing. Each element of the vector can represent a dependence distance or direction. Dependence edges are organized for the user interface using a higher level data abstraction, called the edge list. The edge list provides the user a configurable method of filtering, sorting, and selecting dependences [16, 32].

\subsubsection{Level Vectors}

Dependence edges hold most of the dependence information for a program, but level vectors provide the glue which links them together and to the AST. Every executable statement in a loop nest involved with a dependence has a level vector. A level vector contains entries for each loop nesting level in which the statement is contained. Every entry points to the list of dependences carried at its level. Level vectors therefore provide quick and efficient access to all of the dependences at a particular loop level for each statement. They are used extensively by PED's loop based transformations and user interface.

Because of their high frequency and simplicity, PED treats dependence edges resulting from scalar variables differently. Dependences between a pair of scalar variable references occur for all commonly nested loops. To avoid duplicate edges, only a single edge is stored along with its deepest nesting level. These scalar edges have a separate entry in the level vector. Queries for dependences carried on level $k$ thus cause two entries in the level vector to be searched - the entry at level $k$ and the entry for scalar dependences.

\subsubsection{Reference Lists}

Every variable reference in a loop nest involved in a dependence has a reference list. A reference list points to all dependences that have a given reference as the source or sink. They are useful for transformations such as scalar expansion and array renaming, as well as for reference based user queries.

\subsection{Loop Information and Variable Lists}

Every loop in the program has a structure known as loop info that contains the following information.

- AST pointer for the loop header.

- Level (depth) of the loop in a nest.

- Pointers to the loop info for the previous and next loops.

- Pointers to the shared and private variable lists.

- Flag for parallelized loops.

The shared and private variable lists record the status of all variables accessed in the loop body. Private variables are defined and used only within an iteration of the loop. All other variables are shared. 
Program

do $\mathrm{i}=1,100$

do $\mathrm{j}=1,100$

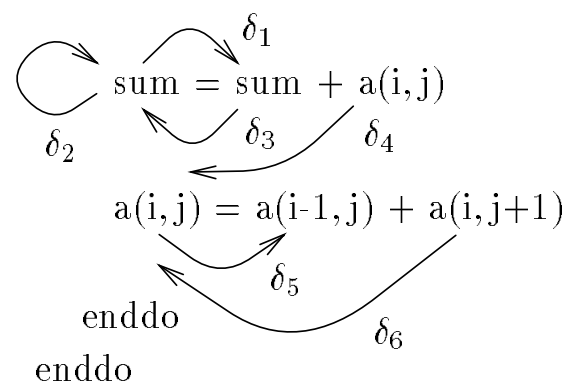

enddo
AST Side Array Info Array

\begin{tabular}{|l|}
\hline loop $:$ do $\mathrm{i}$ \\
\hline loop $:$ do $\mathrm{j}$ \\
\hline stmt $:$ sum $=\ldots$ \\
\hline$i d:$ sum \\
\hline$i d:$ sum \\
\hline subs $: \mathrm{a}(\mathrm{i}, \mathrm{j})$ \\
\hline stmt $: \mathrm{a}(\mathrm{i}, \mathrm{j})=\ldots$ \\
\hline subs $: \mathrm{a}(\mathrm{i}, \mathrm{j})$ \\
\hline subs $: \mathrm{a}(\mathrm{i}-1, \mathrm{j})$ \\
\hline subs $: \mathrm{a}(\mathrm{i}, \mathrm{j}+1)$ \\
\hline
\end{tabular}

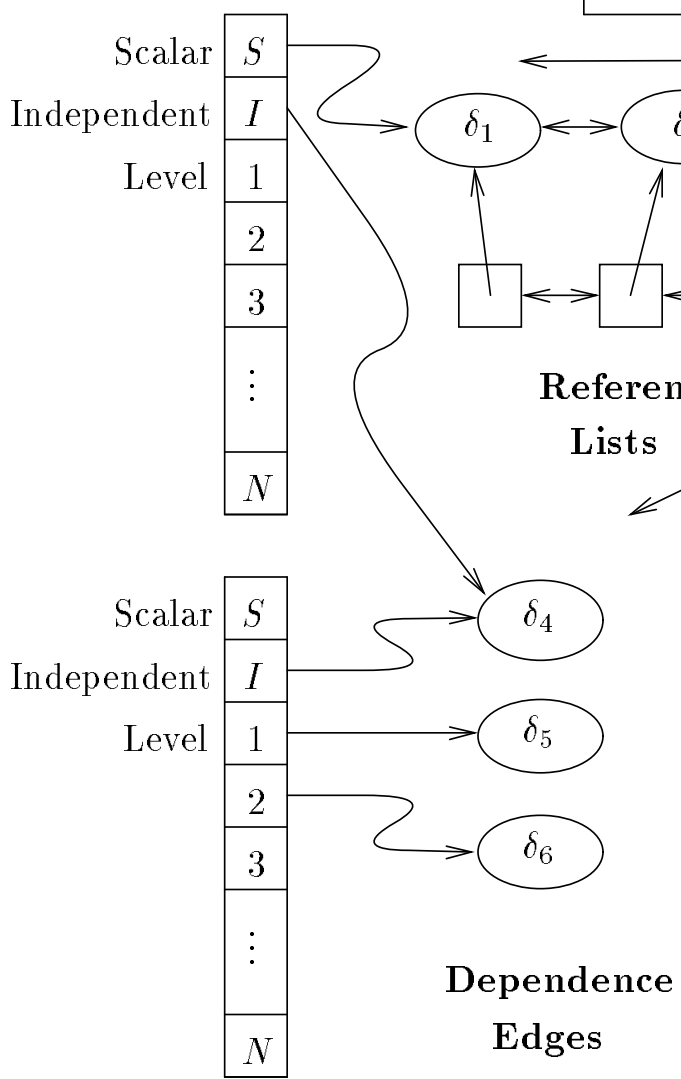

Level

Vectors

\section{Side Array Info Array}
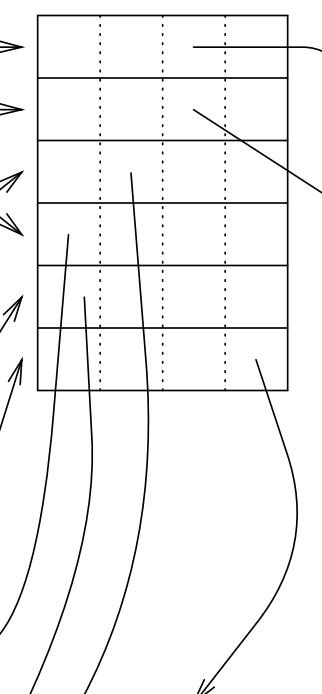
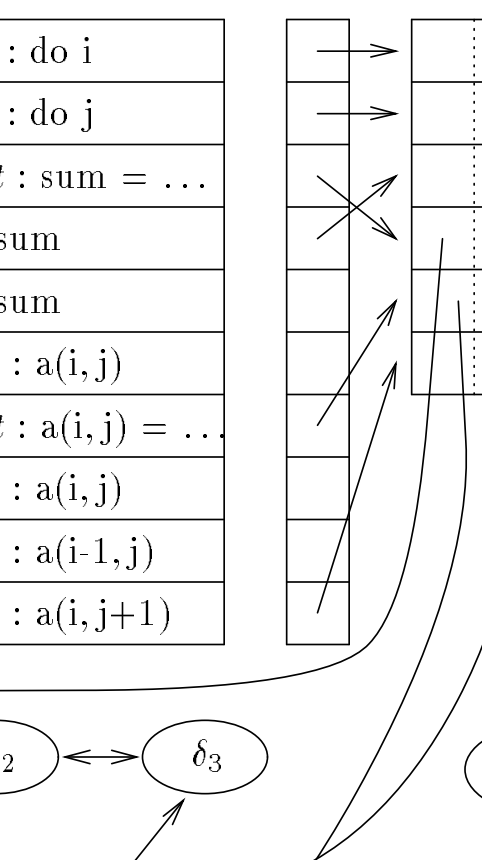
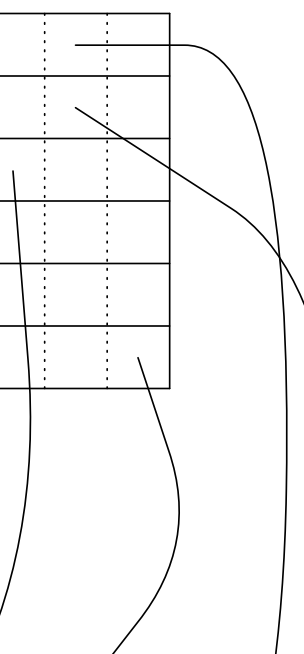


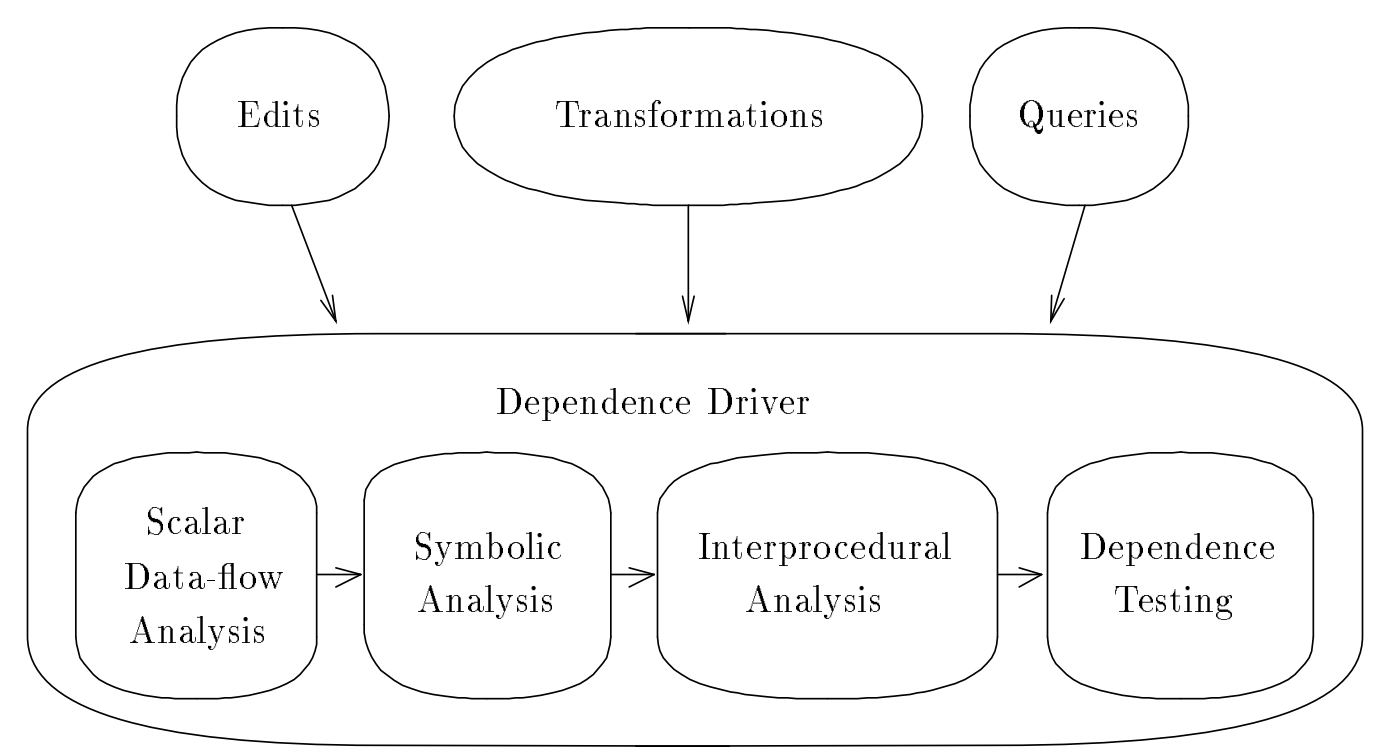

Figure 3: Dependence Analyzer Architecture

\section{Program Analysis}

PED's dependence analyzer faces three major challenges:

Precision Conservative data dependence analysis requires that if a dependence cannot be disproven, it must be assumed to exist. But if these dependences do not actually exist, PED may be inhibited from exploiting the parallelism available in a program. The most important objective of the dependence analyzer is to minimize these false dependences through precise analysis.

Efficiency In order for PED to be truly useful, the dependence analyzer must provide precise results in a reasonable amount of time. PED's dependence analyzer gains efficiency by using fast algorithms for analyzing common simple cases, holding more powerful but expensive algorithms in reserve.

Incrementality Historically, dependence analysis has been performed in batch mode as a single pass through the entire program. In an interactive tool or parallelizing compiler, batch reanalysis after each program transformation has proven to be unacceptably slow. PED's dependence analyzer supports incremental dependence analysis, a technique to limit reanalysis which results in significantly faster updates after program changes. In general, incremental algorithms must be both quick and precise. Otherwise, users will prefer batch analysis. PED achieves efficiency by applying a tiered set of incremental responses based on the scope of the program change. PED's incremental analysis strategy and algorithms are described in Sections 6.1 and 8.1.1.

\subsection{Dependence Analyzer Overview}

The architecture of PED's dependence analyzer is shown in Figure 3. It consists of four major components: the dependence driver, scalar data-flow analysis, symbolic analysis, interprocedural analysis, and dependence testing. The following sections examine each in more detail.

\subsection{Dependence Analysis Driver}

The dependence analysis driver insulates and coordinates the internal phases of the dependence analyzer from the programming environment. It serves four important functions: coordination, query management, 
change notification, and dependence updates.

First, the driver coordinates each of the internal phases of the analyzer. This is especially important for incremental analysis. It also invokes syntax and type checking after edits to ensure that the input program is correct before continuing program analysis. Query management is achieved by hiding the internal representation of the dependence graph and enforcing a standard interface for all queries about dependence information. Queries are insulated from the dependence graph by the driver, allowing lazy dependence updates after a series of edits.

The driver is also responsible for change notification. It receives notice of program changes from the editor and user interface, determines the scope of changes, and decides on an appropriate update strategy. After updates have been performed, the driver must also notify other parts of the environment. Finally, the dependence driver also supports an interface for direct dependence updates. This interface enables efficient incremental updates of dependence information after structured transformations with known effects.

\subsection{Scalar Data-flow Analysis}

Scalar data-flow analysis computes data-flow information, control dependences, and data dependences for scalar variables. It also provides a framework for the later phases of the dependence analyzer. PED first constructs the control flow graph and postdominator tree. It then computes dominance frontiers for each scalar variable and uses them to build the static single assignment (SSA) graph for each procedure [18]. Edges in the SSA graph correspond to precise true dependences for scalar variables.

Next, PED constructs a coarse dependence graph for array variables in each loop nest by connecting $\{$ Defs $\}$ with $\{$ Defs $\cup$ Uses\}. These edges are later refined through dependence testing to construct dependence edges. The same technique is used to build a set of coarse anti and output dependences for scalar variables in loop nests. More precise anti and output dependences may be calculated for scalars using techniques similar to those used in building the SSA graph, but we do not find it necessary. PED also inserts loop-carried dependences for unanalyzed procedure calls, input and output statements, and branches out of loops.

The scalar data-flow analysis phase also calculates information used to determine whether variables in a loop may be safely made private. If a scalar variable is not live outside a specific loop and does not have loop-carried true dependences, it may be made private to eliminate loop-carried storage (anti and output) dependences. Otherwise, the variable is classified as shared. PED currently assumes that all arrays are live outside of loops and are therefore shared. Transformations such as loop distribution also need control dependences, which are calculated from the postdominator tree (see Sections 2.2 and 7.2).

All internal dependence representations and underlying structures are complete in the current implementation of PED. The only remaining task is to compute live ranges and dependences for scalars using the SSA graph.

\subsection{Symbolic Analysis}

Symbolic analysis is the process of representing and evaluating expressions in program variables at compile time. When possible, this phase derives constants or simplified forms for loop bounds, loop steps, array subscript expressions, array dimensions, and control flow. Symbolic analysis significantly improves the precision of dependence testing [20, 22, 44] and interprocedural analysis [24].

Unlike automatic parallelization tools, PED does not make transformations to the program for purposes of analysis. Accordingly, the results of symbolic analysis are stored in shadow expressions that represent 
computations of interest. For example, the dependence testing phase may need to reason about a subscript expression or compare two subscript expressions. The symbolic analyzer will then provide or build an appropriate shadow expression.

The SSA graph for scalars, produced by scalar data-flow analysis, provides the framework for symbolic analysis in PED. Global value numbering is performed on the SSA graph [4]. An extended version of this algorithm simplifies value numbers as they are built, using arithmetic laws and cancellation. Value numbers are then translated into shadow expressions for use by dependence testing and other analyses. Because the value numbering algorithm deduces and propagates constraints and relationships between symbolic expressions, the symbolic analyzer can detect and perform the following:

Constant propagation. Variables and expressions that can be reduced to a constant.

Auxiliary induction variable recognition. Auxiliary induction variables are represented by functions of loop index variables.

Expression folding. Symbolic expressions are simplified and propagated.

Loop-invariant expression recognition. Loop-invariant expressions are identified for use during dependence testing and optimization.

Reduction recognition. Opportunities for using reduction operators are detected.

Value numbering and the resulting shadow expressions have been implemented and incorporated into the dependence tester. Constant propagation, expression folding, loop-invariant expression and reduction recognition are all available. Auxiliary induction variable recognition on the SSA graph is planned. This implementation is part of an ongoing effort that also encompasses interprocedural symbolic analysis.

\subsection{Interprocedural Analysis}

The presence of procedure calls complicates the process of analyzing dependences. Interprocedural analysis is required so that worst case assumptions need not be made when calls are encountered. ParaScope performs conventional interprocedural analysis that discovers constants, aliasing, flow-insensitive side effects such as REF and MOD $[12,17]$. Flow-sensitive side effects such as USE and KILL are not currently available. Even with these analyses, improvements are limited because arrays are treated as monolithic objects, making it impossible to determine whether two references to an array actually access the same memory location.

To provide more precise analysis, array accesses can be summarized in terms of regular sections that describe subsections of arrays such as rows, columns, and rectangles [24]. Local symbolic analysis and interprocedural constants are required to build accurate regular sections. Once constructed, regular sections may be quickly intersected during interprocedural analysis and dependence testing to determine whether dependences exist. The implementation of regular sections in ParaScope is nearing completion. We are also integrating existing ParaScope interprocedural analysis and transformations such as inlining and cloning into PED [17].

\subsection{Dependence Testing}

The dependence testing phase refines the coarse dependence graph for array variables created by scalar analysis and sharpened by interprocedural analysis. PED classifies subscripts in a pair of array references 
according to two orthogonal criteria: complexity and separability. Complexity refers to the number of indices appearing within the subscript. Separability describes whether a given subscript interacts with other subscripts for the purpose of dependence testing.

Using this classification scheme, appropriate dependence tests are selected and applied to each subscript position in a pair of array references. Dependence edges are eliminated if dependence between the references can be disproved. Otherwise, dependence testing characterizes the dependences with a minimal set of hybrid distance/direction vectors. This dependence information is vital for guiding transformations. PED's dependence tests are discussed in detail elsewhere [20]. Most of these dependence tests have been implemented in the current version of PED. We are in the process of extending them to handle symbolic expressions, complex iteration spaces, and regular sections.

\subsection{Analysis of Synchronization}

In a sophisticated parallel program, the user may wish to employ complex synchronization. Typically, synchronization constructs are used to ensure that a dependence is satisfied. When synchronization is present, it is important to eliminate any preserved dependences so that the user will not need to consider them further. Establishing that the order specified by certain dependences will always be observed has been shown to be co-NP-hard, but techniques have been developed to identify dependences that are satisfied by existing synchronization under restricted circumstances [13]. The current implementation of PED can determine if event style synchronization is sufficient to protect a particular dependence.

\subsection{Utilizing External Analysis}

To overcome gaps in the current implementation of program analysis, PED may import dependence information from PFC, the Rice system for automatic vectorization and parallelization [3]. PFC's program analyzer is more mature and contains symbolic analysis, interprocedural regular sections and constants, as well as control and data dependence analysis. PFC produces a file of dependence information that PED converts into its own internal representation. This process is a temporary expedient which will become unnecessary when program analysis in PED is complete.

\section{Transformations}

PED provides a variety of interactive, structured transformations that enhance or expose parallelism in programs. These transformations are applied according to a power steering paradigm: the user specifies the transformation to be made, and the system provides advice and carries out the mechanical details. Thus the user is relieved of the responsibility of making tedious and error prone program changes.

PED evaluates each transformation invoked according to three criteria: applicability, safety, and profitability. A transformation is applicable if it can be mechanically performed. For example, loop interchange is inapplicable for a single loop. A transformation is safe if it preserves the meaning of the original sequential program. Some transformations are always safe, others require a specific dependence pattern. Finally, PED classifies a transformation as profitable if it can determine that the transformation directly or indirectly improves the parallelism of the resulting program.

To perform a transformation, the user makes a program selection and invokes the desired transformation. If the transformation is inapplicable, PED responds with a diagnostic message. If the transformation is safe, PED advises the user as to its profitability. For parameterized transformations, PED may also suggest 
a parameter value. ${ }^{3}$ The user may then apply the transformation.

If the transformation is unsafe or unprofitable, PED responds with a warning explaining the cause. In these cases the user may decide to override the system advice and apply the transformation anyway. For example, if a user decides to parallelize a loop with loop-carried dependences, PED will warn the user of the dependences but allow the loop to be made parallel. This override ability is extremely important in an interactive tool, since it allows the user to apply knowledge unavailable to the tool. The program AST and dependence information are automatically updated after each transformation to reflect the transformed source.

PED supports a large set of transformations that have proven useful for introducing, discovering, and exploiting parallelism. PED also supports transformations for enhancing the use of the memory hierarchy. These transformations are described in detail in the literature [2, 3, 10, 34, 36, 48]. We classify the transformations in PED as follows.

\section{Reordering Transformations}

$\begin{array}{lll}\text { Loop Distribution } & \text { Loop Skewing } & \text { Statement Interchange } \\ \text { Loop Interchange } & \text { Loop Fusion } & \text { Loop Reversal }\end{array}$

Strip Mining

\section{Dependence Breaking Transformations}

$\begin{array}{lll}\text { Privatization } & \text { Scalar Expansion } & \text { Loop Splitting } \\ \text { Array Renaming } & \text { Loop Peeling } & \text { Alignment }\end{array}$

\section{Memory Optimizing Transformations}
Scalar Replacement
Loop Unrolling
Unroll-and-Jam

\section{Miscellaneous Transformations}

Sequential $\leftrightarrow$ Parallel Loop Bounds Adjusting

Statement Addition Statement Deletion

Reordering transformations change the order in which statements are executed, either within or across loop iterations. ${ }^{4}$ They are safe if all program dependences in the original program are preserved. Reordering transformations are used to expose or enhance loop-level parallelism. They are often performed in concert with other transformations to structure computations in a way that allows useful parallelism to be introduced.

Dependence breaking transformations are used to break specific dependences that inhibit parallelism. They may introduce new storage to eliminate storage-related anti or output dependences, or convert loop-carried dependences to loop-independent dependences, often enabling the safe application of other transformations. If all the dependences carried on a loop are eliminated, the loop may then be run in parallel.

Memory optimizing transformations adjust a loop's balance between computations and memory accesses to make better use of the memory hierarchy and functional pipelines. These transformations have proven to be extremely effective for both scalar and parallel machines.

\footnotetext{
${ }^{3}$ For example, see loop skewing and unroll-and-jam in Sections 7.4 and 7.5 .

${ }^{4}$ Loop skewing and strip mining reorder execution only when used in conjunction with loop interchange.
} 


\subsection{Incremental Analysis}

A significant advantage of structured transformations is that their effects are known in advance. In particular, few transformations affect global data-flow or symbolic information. PED can thus perform updates very efficiently. Some transformations may require partial reanalysis, while others may directly update the existing dependence graph. Below, we classify safe transformations based on their PED update algorithms.

None: Statement Interchange, Loop Bounds Adjusting

Move edges: Loop Interchange, Array Renaming

Modify edges: Loop Distribution, Loop Skewing, Loop Reversal, Alignment, Privatization

Delete edges: Scalar Expansion, Statement Deletion

Add edges: Strip Mining, Scalar Replacement

Redo dependence testing: Loop Peeling, Loop Splitting

Redo dependence analysis for loop nest: Loop Fusion, Loop Unrolling, Unroll-and-Jam, Statement Addition

\section{$7 \quad$ Example Transformations}

Although many of the algorithms for applying these transformations have appeared elsewhere, our implementation gives profitability advice and performs incremental updates of dependence information. Rather than describe all these phases for each transformation, we have chosen to examine five interesting transformations in detail. We discuss loop interchange, loop distribution, loop fusion, loop skewing, and unrolland-jam. The purpose, mechanics, and safety of these transformations are presented, followed by their profitability estimates, user advice, and incremental dependence update algorithms.

\section{$7.1 \quad$ Loop Interchange}

Loop interchange is a key transformation that modifies the traversal order of the iteration space for the selected loop nest $[3,48]$. It has been used extensively in vectorizing and parallelizing compilers to adjust the granularity of parallel loops and to expose parallelism [3, 34, 48]. PED interchanges pairs of adjacent loops. Loop permutations may be performed as a series of pairwise interchanges. PED supports interchange of triangular or skewed loops. It also interchanges complex loop nests that result after interchanging skewed loops.

Safety Loop interchange is safe if it does not reverse the order of execution of the source and sink of any dependence. PED determines this by examining the direction vectors for all dependences carried on the outer loop. If any dependence has a direction vector of the form $(<,>)$, interchange is unsafe. These dependences are called interchange-preventing; they are flagged by PED during dependence testing. When applying loop interchange, each dependence edge carried on the outer loop is examined. If any dependence has its interchange-preventing flag set, PED advises the user that interchange is unsafe.

Profitability PED judges the profitability of loop interchange by calculating which of the loops will be parallel after the interchange. A dependence carried on the outer loop will move inward if it has a direction vector of the form $(<,=)$. These dependences are called interchange-sensitive; they are also flagged by PED during dependence testing. When applying loop interchange, PED examines each dependence edge on 
the outer loop to determine where it will be following interchange. It then checks for dependences carried on the inner loop as well; they move outward following interchange. Depending on the result, PED advises the user that neither, one, or both of the loops will be parallel after interchange.

Update Updates after loop interchange are very quick. Dependence edges on the interchanged loops are moved directly to the appropriate loop level based on their interchange-sensitive flags. All the dependences in the loop nest then have the elements in their direction vector corresponding to the interchanged loops swapped, e.g., $(<,=)$ becomes $(=,<)$. Finally, the interchange flags are recalculated for dependences in the loop nest.

\subsection{Loop Distribution}

Loop distribution separates independent statements inside a single loop into multiple loops with identical headers $[3,34]$. It is used to expose partial parallelism by separating statements which may be parallelized from those that must be executed sequentially. Loop distribution is a cornerstone of vectorization and parallelization [3, 34].

In PED the user can specify whether distribution is for the purpose of vectorization or parallelization. If the user specifies vectorization, then each statement is placed in a separate loop when possible. If the user specifies parallelization, then statements are grouped together into the fewest loops such that the most statements can be made parallel. The user is presented with a partition of the statements into new loops, as well as an indication of which loops are parallelizable. The user may then apply or reject the distribution partition.

Safety To maintain the meaning of the original loop, the partition must not put statements that are involved in recurrences into different loops [30,34]. Recurrences are calculated by finding strongly connected regions in the subgraph composed of loop-independent dependences and dependences carried on the loop to be distributed. Statements not involved in recurrences may be placed together or in separate loops, but the order of the resulting loops must preserve all other data and control dependences. PED always computes a partition which meets these criteria.

If there is control flow in the original loop, the partition may cause decisions that occur in one loop to be used in a later loop. These decisions correspond to loop-independent control dependences that cross between partitions. We use Kennedy and McKinley's method to insert new arrays, called execution variables, that record these "crossing" decisions [30]. Given a partition, this algorithm introduces the minimal number of execution variables necessary to effect the partition, even for loops with arbitrary control flow.

Profitability Currently PED does not change the order of statements in the loop during partitioning. This simplification improves the recognizability of the resulting program, but may reduce the parallelism uncovered. In particular, statements that fall lexically between statements in a recurrence will be put into the same partition as the recurrence. In addition, when the source of a dependence lexically follows the sink, these statements will be placed in the same partition. The implementation of a partitioning algorithm that maximizes loop parallelism while producing the fewest parallel loops is underway [31].

When distributing for vectorization, statements not involved in recurrences are placed in separate loops. When distributing for parallelization, they are partitioned as follows. A statement is added to the preceding partition only if it does not cause that partition to be sequentialized. Otherwise it begins a new partition. Consider distributing the left loop below. 


\begin{tabular}{|c|c|c|c|}
\hline DO I $=1, \mathrm{~N}$ & & $S_{1}$ & $\begin{array}{c}\text { PARALLEL DO } \mathrm{I}=1, \mathrm{~N} \\
\mathrm{~A}(\mathrm{i})=\ldots\end{array}$ \\
\hline$A(i)=\ldots$ & $\Longrightarrow$ & & ENDDO \\
\hline$\ldots=\mathrm{A}(\mathrm{i}-1)$ & distribution & & PARALLEL DO $\mathrm{I}=1, \mathrm{~N}$ \\
\hline ENDDO & & $S_{2}$ & $\begin{array}{l}\ldots=\mathrm{A}(\mathrm{i}-1) \\
\text { ENDDO }\end{array}$ \\
\hline
\end{tabular}

This loop contains only the loop-carried true dependence $S_{1} \delta S_{2}$. Since there are no recurrences, $S_{1}$ and $S_{2}$ begin in separate partitions. $S_{1}$ is placed in a parallel partition, then $S_{2}$ is considered. The addition of $S_{2}$ to the partition would instantiate the loop-carried true dependence, causing the partition to be sequential. Therefore, $S_{2}$ is placed in a separate loop and both loops may be made parallel, as illustrated above by the two transformed loops on the right. Loop distribution can also enable loop interchange to enhance parallelism or data locality [3, 14].

Update Updates can be performed quickly on the existing dependence graph after loop distribution. For each new loop PED also creates new loop info structures and attaches them to the AST. Data and control dependences between statements in the same partition remain unchanged. Data dependences carried on the distributed loop between statements placed in separate partitions are converted into loop-independent dependences (as in the above example).

Loop-independent control dependences that cross partitions are deleted and replaced as follows. First, loop-independent data dependences are introduced between the definitions and uses of execution variables representing the crossing decision. A control dependence is then inserted from the test on the execution variable to the sink of the original control dependence. The update algorithm is explained more thoroughly elsewhere [30].

\subsection{Loop Fusion}

Loop fusion is the dual of loop distribution. Instead of breaking up a loop into multiple loops, it combines two loop nests into a single loop nest. Researchers have found loop fusion useful for improving data locality, reducing loop overhead, and enabling loop interchange [14, 31]. PED supports fusion of adjacent loop nests where the outer loops iterate identically, but the loop index variable names need not be the same.

Safety Loop fusion is safe if it does not reverse the order of execution of the source and sink of any dependence [48]. If there are no dependences between two loops, fusion is always safe. If there is a dependence, it is loop-independent. To test if fusion is safe, dependence testing is performed on the loop bodies as if they were in a single loop. A loop-independent dependence between the original nests either remains loop-independent or becomes forward loop-carried or backward loop-carried in the fused loop. If it remains loop-independent, fusion is safe. If it becomes forward loop-carried, fusion is safe but may reduce parallelism. If it becomes backward loop-carried, fusion is not safe because the flow of values would be reversed.

Profitability Loop fusion is profitable if it increases the granularity of parallelism by fusing two parallel loops, since fusion eliminates a synchronization point. PED will warn the user if parallelism would be lost after fusion, either by creating new loop-carried dependences or by fusing sequential and parallel loops. 

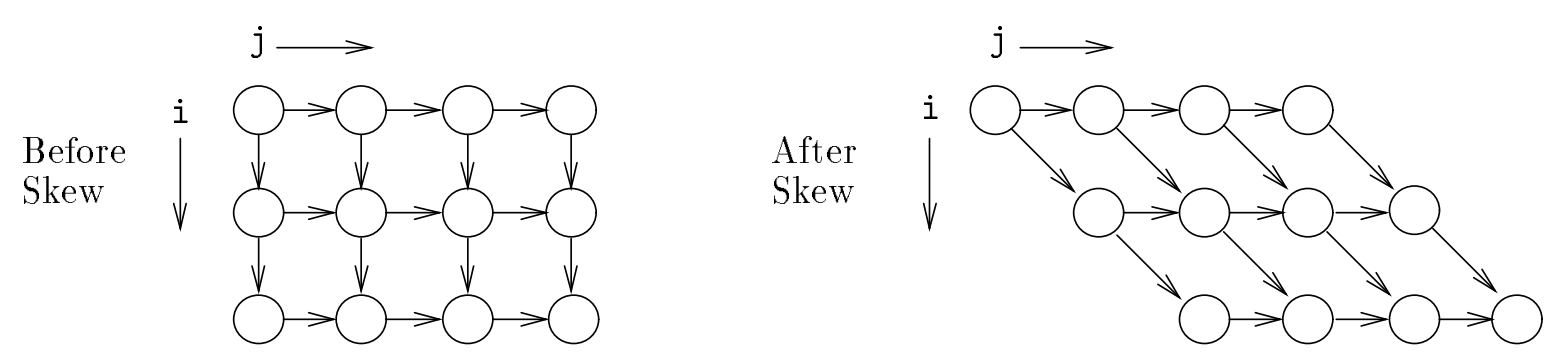

Figure 4: Effect of Loop Skew on Dependences and Iteration Space

Loop fusion can also improve data locality by bring two references closer together in time. Consider the example below.

$$
\begin{array}{r}
\mathrm{DO} I=1, \mathrm{~N} \\
\mathrm{~A}(\mathrm{I})=\mathrm{I}
\end{array}
$$

ENDDO

DO I $=1, \mathrm{~N}$

ENDDO

$$
\begin{aligned}
& \text { DO I }=1, \mathrm{~N} \\
& \mathrm{~A}(\mathrm{I})=\mathrm{I} \\
& \mathrm{B}(\mathrm{I})=\mathrm{A}(\mathrm{I}) * \mathrm{~A}(\mathrm{I}) \\
& \text { ENDDO }
\end{aligned}
$$

In the version on the left, the write to A(I) occurs N iterations before the subsequent reads. After fusion, the write and the read occur on the same iteration, making A(I) much more likely to still be in cache or a register at the time of the read. Loop fusion can also improve data locality and increase parallelism by enabling loop interchange [14, 31]. PED does not currently report these cases.

Update Updates after loop fusion are straightforward and quick. The loop info structures are merged. If the loop index variables differ they are both replaced with a new unique name. The control dependences on the original loop headers are adjusted to the new loop header. Because no global data-flow or symbolic information is changed by fusion, PED simply rebuilds the scalar dependence graph for the new loop nest and refines it with dependence tests. This update strategy proved simple to implement and is quick in practice.

\subsection{Loop Skewing}

Loop skewing is a transformation that changes the shape of the iteration space to expose parallelism across a wavefront $[29,48]$. It can be applied via unimodular methods using loop interchange, strip mining, and loop reversal to obtain loop-level parallelism in a loop nest [7, 47]. All of these transformations are supported in PED.

Loop skewing is applied to a pair of perfectly nested loops that both carry dependences, even after loop interchange. Loop skewing adjusts the iteration space of these loops by shifting the work per iteration, changing the shape of the iteration space from a rectangle to a parallelogram. Figure 4 illustrates an iteration space before and after skewing. Skewing changes dependence distances for the inner loop so that all dependences are carried on the outer loop after loop interchange. The inner loop can then be safely parallelized.

Loop skewing of degree $\alpha$ is performed by adding $\alpha$ times the outer loop index variable to the upper and lower bounds of the inner loop, followed by subtracting the same amount from each occurrence of the inner loop index variable in the loop body. In the example below, the second loop nest results when the J loop in the first loop nest is skewed by degree 1 with respect to loop I. 


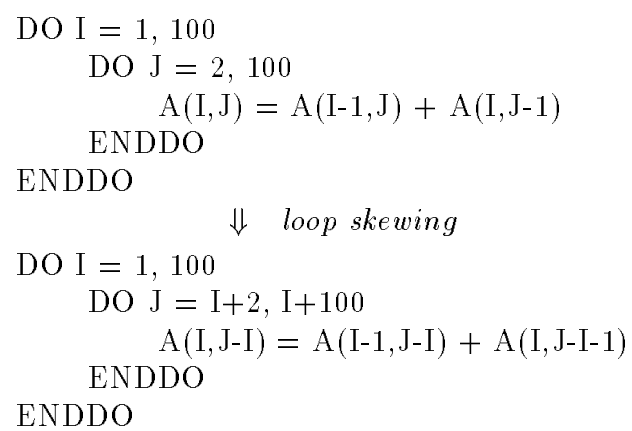

Figure 4 illustrates the iteration space for this example. For the original loop, dependences with distance vectors $(1,0)$ and $(0,1)$ prevent either loop from being safely parallelized. In the skewed loop, the distance vectors for dependences are transformed to $(1,1)$ and $(0,1)$. There are no longer any dependences within each column of the iteration space, so parallelism is exposed. However, to introduce the parallelism on the I loop requires also performing loop interchange.

Safety Loop skewing is always safe because it does not change the order in which array memory locations are accessed. It only changes the shape of the iteration space.

Profitability To determine if skewing is profitable, PED ascertains whether skewing will expose parallelism that can be made explicit using loop interchange and suggests the minimum skew amount needed to do so. This analysis requires that all dependences carried on the outer loop have precise distance vectors. Skewing is only profitable if:

1. $\exists$ dependences on the inner loop, and

2. $\exists$ at least one dependence on the outer loop with a distance vector $\left(d_{1}, d_{2}\right)$, where $d_{2} \leq 0$.

The interchange-preventing or interchange-sensitive dependences in (2) prevent the application of loop interchange to move all dependences to the outer loop. If they do not exist, at least one loop may already be safely parallelized, possibly by using loop interchange. The purpose of loop skewing is to change the distance vector to $\left(d_{1}, d_{2}^{\prime}\right)$, where $d_{2}^{\prime} \geq 1$. In terms of the iteration space, loop skewing is needed to transform dependences that point down or downwards to the left into dependences that point downwards to the right. Followed by loop interchange, these dependences will remain on the outer loop, allowing the inner loop to be safely parallelized.

To compute the skew degree, we first consider the effect of loop skewing on each dependence. When skewing the inner loop with respect to the outer loop by an integer degree $\alpha$, the original distance vector $\left(d_{1}, d_{2}\right)$ becomes $\left(d_{1}, \alpha d_{1}+d_{2}\right)$. So for any dependence where $d_{2} \leq 0$, we want $\alpha$ such that $\alpha d_{1}+d_{2} \geq 1$. To find the minimal skew degree we compute

$$
\alpha=\left\lceil\frac{1-d_{2}}{d_{1}}\right\rceil
$$

for each dependence, taking the maximum $\alpha$ for all the dependences; this is suggested as the skew degree. Update Updates after loop skewing are also very fast. After skewing by degree $\alpha$, the incremental update algorithm changes the original distance vectors $\left(d_{1}, d_{2}\right)$ for all dependences in the nest to $\left(d_{1}, \alpha d_{1}+d_{2}\right)$, and then updates their interchange flags. 


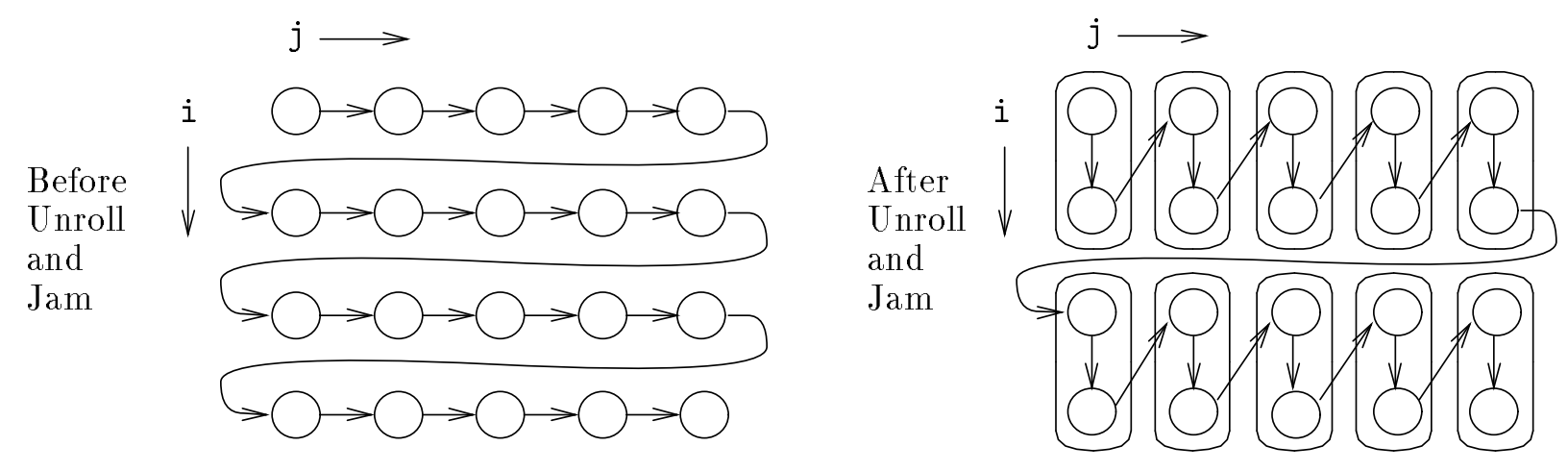

Figure 5: Effect of Unroll-and-Jam on Iteration Space

\subsection{Unroll-and-Jam}

Unroll-and-jam is a transformation that unrolls an outer loop in a loop nest, then jams (or fuses) the resulting inner loops $[2,11]$. Unroll-and-jam can be used to convert dependences carried by the outer loop into loop independent dependences or dependences carried by some inner loop. It brings two accesses to the same memory location closer together and can significantly improve performance by enabling reuse of either registers or cache. When applied in conjunction with scalar replacement on scientific codes, unrolland-jam has resulted in integer factor speedups, even for single processors [10]. Unroll-and-jam may also be applied to imperfectly nested loops or loops with complex iteration spaces. Figure 5 shows an example iteration space before and after unroll-and-jam of degree 1.

Before performing unroll-and-jam of degree $\alpha$ on a loop with step $\sigma$, we may need to use loop splitting to make the total number of iterations divisible by $\alpha+1$ by separating the first few iterations of the loop into a preloop. We then create $\alpha$ additional copies of the loop body. All occurrences of the loop index variable in the $i^{\text {th }}$ new loop body must be incremented by $\sigma i$. The step of the loop is then increased to $\sigma(\alpha+1)$.

In the following matrix multiply example, loop I is unrolled and jammed by one to bring together references to $\mathrm{B}(\mathrm{K}, \mathrm{J})$, resulting in the second loop nest.

$\mathrm{DO} \mathrm{I}=1,100$

DO $\mathrm{J}=1,100$

$\mathrm{C}(\mathrm{I}, \mathrm{J})=0.00$

DO $\mathrm{K}=1,100$

ENDDO

$\mathrm{C}(\mathrm{I}, \mathrm{J})=\mathrm{C}(\mathrm{I}, \mathrm{J})+\mathrm{A}(\mathrm{I}, \mathrm{K}) * \mathrm{~B}(\mathrm{~K}, \mathrm{~J})$

ENDDO

ENDDO

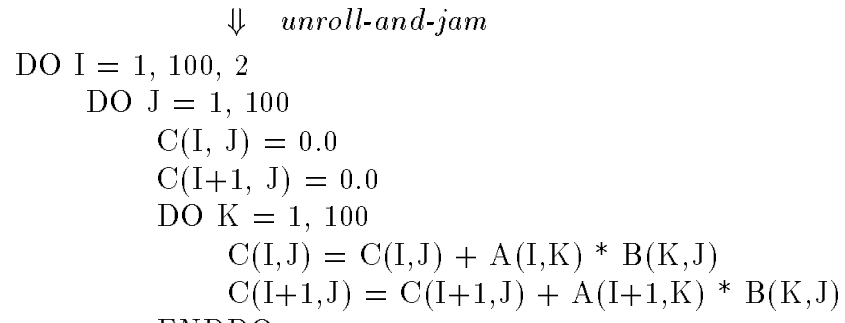

ENDDO

ENDDO

Unroll-and-jam could also be performed on loop J to bring together references to A(I,K). 
Safety To determine safety, an alternative formulation of unroll-and-jam is used. Unroll-and-jam is equivalent to strip mining the outer loop by the unroll degree, interchanging the strip mined loop to the innermost position, and then completely unrolling the strip mined loop. Since strip mining and loop unrolling are always safe, we only need to determine whether we can safely interchange the strip mined loop to the innermost position.

PED determines this by searching for interchange-preventing dependences on the outer loop. Unrolland-jam is unsafe if any dependence carried by the outer loop has a direction vector of the form $(<,>)$. Even if such a dependence is found, unroll-and-jam is still safe if the unroll degree is less than the distance of the dependence on the outer loop, since this dependence would remain carried by the outer loop. PED will either warn the user that unroll-and-jam is unsafe, or provide a range of safe unroll degrees.

Unroll-and-jam of imperfectly nested loops changes the execution order of the imperfectly nested statements with respect to the rest of the loop body. Dependences carried on the unrolled loop with distance less than or equal to the unroll degree are converted into loop-independent dependences. If any of these dependences cross between the imperfectly nested statements and the statements in the inner loop, they inhibit unroll-and-jam. Specifically, the intervening statements cannot be moved and prevent fusion of the inner loops.

Profitability Balance describes the ratio between computation and memory access rates [11]. Unrolland-jam is profitable if it brings the balance of a loop closer to the balance of the underlying machine. PED automatically calculates the optimal unroll-and-jam degree for a loop nest, including loops with complex iteration spaces [10].

Update An algorithm for the incremental update of the dependence graph after unroll-and-jam is described elsewhere [10]. However, since no global data-flow or symbolic information is changed by unrolland-jam, we chose the same strategy as for loop fusion. PED simply rebuilds the scalar dependence graph for the loop nest and applies dependence tests to the resulting edges.

\subsection{Implementation Status}

All of the structured program transformations in this paper have been implemented in PED, including their corresponding incremental update algorithms. We are, as always, in the process of further extending PED's transformation capabilities. An effort is underway to incorporate automatic parallelization strategies within PED in order to provide users with further assistance in the parallelization process [23, 37].

\section{Edits}

This section describes strategies for updates after edits to the program text or direct modification of PED's dependence information. We refer to the first as program edits and the latter as dependence edits.

\subsection{Program Edits}

Editing is fundamental for any program development tool because it is the most flexible means of making program changes. The ParaScope Editor therefore provides advanced editing features. When editing, the user has complete access to the functionality of the hybrid (text and structure) editor underlying PED, including simple text entry, template-based editing, search and replace functions, intelligent and customizable view filters, and automatic syntax and type checking.

Rather than reanalyze immediately after each edit, PED waits for a reanalyze command from the user. This avoids analyzing intermediate stages of the program that may be illegal or simply uninteresting to 
the user. Both transformations and the dependence display are disabled during an editing session, because they rely on dependence information that may be invalidated by the edits. Once the user prompts PED, the dependence driver invokes syntax and type checking. If errors are detected, the user is warned. Otherwise reanalysis proceeds.

\subsubsection{Incremental Analysis}

Unfortunately, incremental dependence analysis after edits is a very difficult problem. As we have already seen, precise dependence analysis requires utilization of several different kinds of information. In order to calculate precise dependence information, PED may need to incrementally update the control flow, control dependence, SSA, and call graphs, as well as recalculate live range, constant, symbolic, interprocedural, and dependence testing information.

Several algorithms for incremental analysis can be found in the literature; e.g., data-flow analysis [41], interprocedural analysis [8], interprocedural recompilation analysis [9], as well as dependence analysis [40]. However, few of these algorithms have been implemented and evaluated in an interactive environment. Rather than tackle all these problems at once, we chose a simple yet practical strategy for the current implementation of PED. First, the scope of each program change is evaluated. Incremental analysis is applied only when it may be profitable, otherwise batch dependence analysis is invoked. PED will apply incremental dependence analysis when the following situations are detected:

No update needed Many program edits fall into this category. It is trivial to determine that changes to comments or whitespace do not require reanalysis. Other cases include changes to arithmetic expressions that do not disturb control flow or symbolic analysis. For instance, changing the assignment $A(I)=B(I)$ to $\mathrm{A}(\mathrm{I})=\mathrm{B}(\mathrm{I})+1$ does not affect dependence information one whit.

Delete dependence edges Removal of an array reference may be handled simply by deleting all edges involving that reference.

Add dependence edges Addition of an array reference may be handled by scanning the loop nest for occurrences of the same variable, performing dependence tests between the new reference and any other references, and adding the necessary dependence edges.

Redo dependence testing Changes to loop bounds or array subscript expressions require dependence testing to be performed on all affected array variables.

Redo local symbolic analysis Some types of program changes do not affect the scalar dependence graph, but may require symbolic analysis to be reapplied. For instance, changing the assignment J=J+1 to $\mathrm{J}=\mathrm{J}+2$, where $\mathrm{J}$ is an auxiliary induction variable, requires redoing symbolic analysis and dependence testing.

Redo local dependence analysis Changes such as the modification of control flow or variables involved in symbolic analysis require significant updates best handled by redoing dependence analysis. However, the nature of the change may allow the reanalysis to be limited to the current loop nest or procedure. In these cases, the entire program does not need to be reanalyzed.

\subsubsection{Implementation Status}

Editing is fully supported in PED, but difficulties with the underlying editor currently require batch dependence analysis to be performed at the end of an editing session. However, the incremental framework is in place. 


\subsection{Dependence Edits}

In PED, users are given the opportunity to change the program through edits and structured transformations. In addition, users may directly change the results of program analysis by modifying the dependence graph or the classification of variables as shared or private. This functionality is needed because program analysis is necessarily conservative. PED's user interface provides a mechanism that enables users to reject a particular dependence or a class of dependences that they feel are overly conservative $[6,16,23,32]$. In response, PED no longer considers these dependences during program transformations. Users can similarly correct overly conservative variable classifications by reclassifying shared variables as private. In response, PED eliminates from further consideration any loop-carried dependences incident on these variables. These user assertions are retained even after structured transformations and edits. However, if a change results in batch reanalysis, any dependence and variable assertions will be lost. Plans are in place to provide facilities in PED for more persistent user assertions [23].

\section{Modular Design}

The ParaScope Editor is designed so that its analysis and transformation capabilities can be used throughout the ParaScope programming environment [16]. By separating the calculation of safety and profitability for program transformations, we have made them easily adaptable by other tools. Transformation are easily integrated into new systems by using PED's tests for legality and substituting new profitability measures. This feature has proven itself to be extremely valuable in practice. The analysis and transformation features of PED are currently being used by the Fortran D compiler for distributed-memory machines [26], a source-to-source Fortran translator for improving data locality [10, 14], and an on-the-fly data-race detection system for shared-memory machines [27].

\section{Related Work}

Several other research groups are also developing advanced parallel programming tools. PED's analysis and transformation capabilities compare favorably to automatic parallelization systems such as Parafrase, Ptran, Pips and of course Pfc. Our work on interactive parallelization bears similarities to Ptool, Sigmacs, Pat, Superb, Tiny, and Forge 90.

PED has been greatly influenced by the Rice Parallel Fortran Converter (PFC), which has focused on the problem of automatically vectorizing and parallelizing sequential Fortran [3]. PFC has a mature dependence analyzer which performs data dependence analysis, control dependence analysis, interprocedural constant propagation [12], interprocedural side effect analysis of scalars [17], and interprocedural array section analysis [24]. The precursor to PED, a dependence browser named PTOOL, interactively displayed PFC's dependences to users [25]. Ped integrates and extends PFC's analysis \& transformations and Ptool's browsing capabilities, making them available to the user in an interactive environment.

PARAFRASE was the first automatic vectorizing and parallelizing compiler [34]. It supports program analysis and performs a large number of program transformations to improve parallelism. In PARAFRASE, program transformations are structured in phases and are always applied where applicable. Batch analysis is performed after each transformation phase to update the dependence information for the entire program. PARAFRASE-2 adds scheduling, improved program analysis, and transformations [38]. More advanced interprocedural and symbolic analysis is planned [22]. PARAfRASE-2 uses FAUST as a front end to provide interactive parallelization and graphical displays [21]. 
PTRAN is also an automatic parallelizer with extensive program analysis [1]. It computes the SSA and program dependence graphs, and performs constant propagation and interprocedural analysis [19]. PTRAN introduces both task and loop parallelism, but currently the only other program transformations are variable privatization and loop distribution. Sarkar and Thekkath propose a unified framework for applying iteration-reordering transformations to perfect loop nests [42]. The framework provides rules for calculating legality and updating dependence vectors and loop bounds, but does not estimate profitability.

PIPS applies sophisticated interprocedural semantical analysis to parallelize programs for shared and distributed-memory machines [28]. The user can select the precision of analysis desired, inspect the resulting predicates and regions calculated by PIPS, and then apply transformations such as privatization, loop distribution, and parallelization to specified procedures or the entire program.

SIGMACS, a programmable interactive parallelizer in the FAUST programming environment, computes and displays call graphs, process graphs, and a statement dependence graph [21, 43]. In a process graph each node represents a task or a process, which is a separate entity running in parallel. The call and process graphs may be animated dynamically at run time. Sigmacs also performs several interactive program transformations, and is working on automatic updating of dependence information.

PAT is also an interactive parallelization tool [45]. Its dependence analysis is restricted to Fortran programs where only one write occurs to each variable in a loop. PAT supports replication and alignment, insertion and deletion of assignment statements, and loop parallelization. It can also insert synchronization to protect specific dependences. PAT divides analysis into scalar and dependence phases, but does not perform symbolic or interprocedural analysis. The incremental dependence update that follows transformations is simplified due to its austere analysis [46].

Superb interactively converts sequential programs into data parallel SPMD programs that can be executed on the SuPRENum distributed memory multiprocessor [50]. Super B provides a set of interactive program transformations, including transformations that exploit data parallelism. The user specifies a data partitioning, then node programs with the necessary send and receive operations are automatically generated. Algorithms are also described for incremental update of use-def and def-use chains following structured program transformations [33].

TINY provides precise data dependence analysis and program transformations for a core subset of Fortran [49]. It is particularly adept at performing complex loop transformations on imperfectly nested loops. Extensions to TINY include precise dependence tests, array kill analysis, and automatic selection of program transformation sequences [39].

Forge 90, formerly Mimdizer, is an interactive parallelization system for MIMD shared and distributed-memory machines from Applied Parallel Research [5]. It performs data-flow and dependence analyses, and also supports loop-level transformations. Associated tools graphically display call graph, control flow, dependence, and profiling information. Forge 90 can be used to generate parallel programs for both shared and distributed-memory machines. Computer vendors such as Cray Research Inc. and Silicon Graphics Inc. have also begun providing interactive parallel programming tools similar to PED.

\section{Conclusions}

Our experience with the ParaScope Editor has shown that dependence analysis can be used in an interactive tool with acceptable efficiency. This efficiency is due to fast yet precise dependence analysis algorithms, and a dependence representation that makes it easy to find dependences and to reconstruct them after a 
change. To our knowledge, PED is the first tool to offer general editing with dependence reconstruction along with a substantial collection of useful program transformations.

PED's ability to analyze and display dependence information has made it into a powerful tool for the experienced parallel programmer. By reviewing the dependences carried by a particular parallel loop, the programmer can avoid the kinds of simple mistakes that may later take months to find and correct. In this paper we have shown how to keep the cost of providing this information low enough to make PED a practical interactive tool. By separating the calculation of safety and profitability, we have also made PED's transformation abilities easily available to other tools.

\section{Acknowledgments}

We wish to thank Paul Havlak both for his assistance on this paper and for his role as the principal architect of scalar and symbolic analysis in PED. We gratefully acknowledge Alan Carle, Mary Hall, Nat McIntosh, John Mellor-Crummey, Mike Paleczny, Hariklia Tsalapatas and Scott Warren for their valuable contributions to the current ParaScope implementation. We are indebted to the PFC, $\mathbb{R}^{\mathrm{n}}$, and ParaScope research groups past and present for providing the software infrastructure upon which PED is built. The efforts of all these people have made PED the useful research tool it is today.

\section{References}

[1] F. Allen, M. Burke, P. Charles, R. Cytron, and J. Ferrante. An overview of the PTRAN analysis system for multiprocessing. In Proceedings of the First International Conference on Supercomputing. Springer-Verlag, Athens, Greece, June 1987.

[2] F. Allen and J. Cocke. A catalogue of optimizing transformations. In J. Rustin, editor, Design and Optimization of Compilers. Prentice-Hall, 1972.

[3] J. R. Allen and K. Kennedy. Automatic translation of Fortran programs to vector form. ACM Transactions on Programming Languages and Systems, 9(4):491-542, October 1987.

[4] B. Alpern, M. Wegman, and K. Zadeck. Detecting equality of variables in programs. In Proceedings of the Fifteenth Annual ACM Symposium on the Principles of Programming Languages, San Diego, CA, January 1988.

[5] Applied Parallel Research, Placerville, CA. Forge 90 Distributed Memory Parallelizer: User's Guide, version 8.0 edition, 1992.

[6] V. Balasundaram, K. Kennedy, U. Kremer, K. S. McKinley, and J. Subhlok. The ParaScope Editor: An interactive parallel programming tool. In Proceedings of Supercomputing '89, Reno, NV, November 1989.

[7] U. Banerjee. Unimodular transformations of double loops. In Advances in Languages and Compilers for Parallel Computing, Irvine, CA, August 1990. The MIT Press.

[8] M. Burke. An interval-based approach to exhaustive and incremental interprocedural data-flow analysis. $A C M$ Transactions on Programming Languages and Systems, 12(3):341-395, July 1990.

[9] M. Burke and L. Torczon. Interprocedural optimization: Eliminating unnecessary recompilation. ACM Transactions on Programming Languages and Systems, to appear 1993.

[10] D. Callahan, S. Carr, and K. Kennedy. Improving register allocation for subscripted variables. In Proceedings of the SIGPLAN '90 Conference on Program Language Design and Implementation, White Plains, NY, June 1990.

[11] D. Callahan, J. Cocke, and K. Kennedy. Estimating interlock and improving balance for pipelined machines. Journal of Parallel and Distributed Computing, 5(4):334-358, August 1988. 
[12] D. Callahan, K. Cooper, K. Kennedy, and L. Torczon. Interprocedural constant propagation. In Proceedings of the SIGPLAN '86 Symposium on Compiler Construction, Palo Alto, CA, June 1986.

[13] D. Callahan, K. Kennedy, and J. Subhlok. Analysis of event synchronization in a parallel programming tool. In Proceedings of the Second ACM SIGPLAN Symposium on Principles and Practice of Parallel Programming, Seattle, WA, March 1990.

[14] S. Carr, K. Kennedy, K. S. McKinley, and C. Tseng. Compiler optimizations for improving data locality. Technical Report TR92-195, Dept. of Computer Science, Rice University, November 1992.

[15] D. Chen, H. Su, and P. Yew. The impact of synchronization and granularity on parallel systems. In Proceedings of the 17th International Symposium on Computer Architecture, Seattle, WA, May 1990.

[16] K. Cooper, M. W. Hall, R. T. Hood, K. Kennedy, K. S. McKinley, J. M. Mellor-Crummey, L. Torczon, and S. K. Warren. The ParaScope parallel programming environment. Proceedings of the IEEE, To appear 1993.

[17] K. Cooper, K. Kennedy, and L. Torczon. The impact of interprocedural analysis and optimization in the $\mathbb{R}^{\mathrm{n}}$ programming environment. ACM Transactions on Programming Languages and Systems, 8(4):491-523, October 1986.

[18] R. Cytron, J. Ferrante, B. Rosen, M. Wegman, and K. Zadeck. Efficiently computing static single assignment form and the control dependence graph. ACM Transactions on Programming Languages and Systems, 13(4):451490, October 1991.

[19] J. Ferrante, K. Ottenstein, and J. Warren. The program dependence graph and its use in optimization. ACM Transactions on Programming Languages and Systems, 9(3):319-349, July 1987.

[20] G. Goff, K. Kennedy, and C. Tseng. Practical dependence testing. In Proceedings of the SIGPLAN'91 Conference on Program Language Design and Implementation, Toronto, Canada, June 1991.

[21] V. Guarna, D. Gannon, Y. Gaur, and D. Jablonowski. Faust: An environment for programming parallel scientific applications. In Proceedings of Supercomputing '88, Orlando, FL, November 1988.

[22] M. Haghighat and C. Polychronopoulos. Symbolic dependence analysis for high-performance parallelizing compilers. In Advances in Languages and Compilers for Parallel Computing, Irvine, CA, August 1990. The MIT Press.

[23] M. W. Hall, T. Harvey, K. Kennedy, N. McIntosh, K. S. McKinley, J. D. Oldham, M. Paleczny, and G. Roth. Experiences using the ParaScope Editor. In Proceedings of the Fourth ACM SIGPLAN Symposium on Principles and Practice of Parallel Programming, San Diego, CA, May 1993.

[24] P. Havlak and K. Kennedy. An implementation of interprocedural bounded regular section analysis. IEEE Transactions on Parallel and Distributed Systems, 2(3):350-360, July 1991.

[25] L. Henderson, R. Hiromoto, O. Lubeck, and M. Simmons. On the use of diagnostic dependency-analysis tools in parallel programming: Experiences using PTOOL. The Journal of Supercomputing, 4:83-96, 1990.

[26] S. Hiranandani, K. Kennedy, and C. Tseng. Compiling Fortran D for MIMD distributed-memory machines. Communications of the ACM, 35(8):66-80, August 1992.

[27] R. Hood, K. Kennedy, and J. Mellor-Crummey. Parallel program debugging with on-the-fly anomaly detection. In Proceedings of Supercomputing '90, New York, NY, November 1990.

[28] F. Irigoin, P. Jouvelot, and R. Triolet. Semantical interprocedural parallelization: An overview of the PIPS project. In Proceedings of the 1991 ACM International Conference on Supercomputing, Cologne, Germany, June 1991.

[29] F. Irigoin and R. Triolet. Supernode partitioning. In Proceedings of the Fifteenth Annual ACM Symposium on the Principles of Programming Languages, San Diego, CA, January 1988.

[30] K. Kennedy and K. S. McKinley. Loop distribution with arbitrary control flow. In Proceedings of Supercomputing 
'90, New York, NY, November 1990.

[31] K. Kennedy and K. S. McKinley. Maximizing loop parallelism and improving data locality via loop fusion and distribution. Technical Report TR92-189, Dept. of Computer Science, Rice University, August 1992.

[32] K. Kennedy, K. S. McKinley, and C. Tseng. Interactive parallel programming using the ParaScope Editor. IEEE Transactions on Parallel and Distributed Systems, 2(3):329-341, July 1991.

[33] U. Kremer, H. Zima, H.-J. Bast, and M. Gerndt. Advanced tools and techniques for automatic parallelization. Parallel Computing, 7:387-393, 1988.

[34] D. Kuck, R. Kuhn, D. Padua, B. Leasure, and M. J. Wolfe. Dependence graphs and compiler optimizations. In Conference Record of the Eighth Annual ACM Symposium on the Principles of Programming Languages, Williamsburg, VA, January 1981.

[35] B. Leasure, editor. PCF Fortran: Language Definition, version 3.1. The Parallel Computing Forum, Champaign, IL, August 1990.

[36] D. Loveman. Program improvement by source-to-source transformations. Journal of the ACM, 17(2):121-145, January 1977 .

[37] K. S. McKinley. Automatic and Interactive Parallelization. PhD thesis, Rice University, April 1992.

[38] C. Polychronopoulos, M. Girkar, M. Haghighat, C. Lee, B. Leung, and D. Schouten. The structure of Parafrase2: An advanced parallelizing compiler for C and Fortran. In D. Gelernter, A. Nicolau, and D. Padua, editors, Languages and Compilers for Parallel Computing. The MIT Press, 1990.

[39] W. Pugh and D. Wonnacott. Eliminating false data dependences using the Omega test. In Proceedings of the SIGPLAN '92 Conference on Program Language Design and Implementation, San Francisco, CA, June 1992.

[40] C. Rosene. Incremental Dependence Analysis. PhD thesis, Rice University, March 1990.

[41] B. Ryder and M. Paull. Incremental data flow analysis algorithms. ACM Transactions on Programming Languages and Systems, 10(1):1-50, January 1988.

[42] V. Sarkar and R. Thekkath. A general framework for interation-reordering loop transformations (technical summary). In Proceedings of the SIGPLAN'92 Conference on Program Language Design and Implementation, San Francisco, CA, June 1992.

[43] B. Shei and D. Gannon. SIGMACS: A programmable programming environment. In Advances in Languages and Compilers for Parallel Computing, Irvine, CA, August 1990. The MIT Press.

[44] J. Singh and J. Hennessy. An empirical investigation of the effectiveness of and limitations of automatic parallelization. In Proceedings of the International Symposium on Shared Memory Multiprocessors, Tokyo, Japan, April 1991.

[45] K. Smith and W. Appelbe. PAT - an interactive Fortran parallelizing assistant tool. In Proceedings of the 1988 International Conference on Parallel Processing, St. Charles, IL, August 1988.

[46] K. Smith, W. Appelbe, and K. Stirewalt. Incremental dependence analysis for interactive parallelization. In Proceedings of the 1990 ACM International Conference on Supercomputing, Amsterdam, The Netherlands, June 1990.

[47] M. E. Wolf and M. Lam. A loop transformation theory and an algorithm to maximize parallelism. IEEE Transactions on Parallel and Distributed Systems, 2(4):452-471, October 1991.

[48] M. J. Wolfe. Optimizing Supercompilers for Supercomputers. The MIT Press, Cambridge, MA, 1989.

[49] M. J. Wolfe. The Tiny loop restructuring research tool. In Proceedings of the 1991 International Conference on Parallel Processing, St. Charles, IL, August 1991.

[50] H. Zima, H.-J. Bast, and M. Gerndt. SUPERB: A tool for semi-automatic MIMD/SIMD parallelization. Parallel Computing, 6:1-18, 1988 . 